\title{
Estratificação de Risco Cardiovascular na Atenção Primária segundo Escore de Framingham ${ }^{1 .}$
}

\section{Cardiovascular Risk Stratification in the Primary Care according to Framingham's Score.}

\section{Estratificación del riesgo cardiovascular en la atención primaria de acuerdo a Framingham Score.}

\author{
Naira Pereira de SOUSA ${ }^{2}$ \\ Maria Fátima de SOUSA ${ }^{3}$ \\ Djane Elys Rene de ARAÚJO ${ }^{4}$ \\ Walterlânia Silva SANTOS 5 \\ Luciano Ramos de LIMA ${ }^{6}$ \\ Tania Cristina Morais Santa Barbara REHEM ${ }^{7}$
}

RESUMO: Objetivo: Estratificar o risco cardiovascular em pacientes portadores de hipertensão arterial sistêmica, atendidos em Regional de Saúde no Distrito Federal. Método: Estudo de abordagem quantitativa, com amostra constituída por 51 usuários. Foi aplicado à amostra o Escore de Risco de Framingham que se baseia nos seguintes fatores preditores: idade, LDL (Low Density Lipoproteins) colesterol, HDL (High Density Lipoproteins) colesterol, pressão arterial, diabetes e tabagismo. Os dados foram analisados por meio de média aritmética, desvio-padrão, frequência absolutas e relativas. Resultados: $43,1 \%$ apresentou baixo risco, 41,2\% moderado risco e 15,7\% alto risco cardiovascular. Conclusão: Apesar do Escore de Risco de Framingham ser mais utilizado em cenários externos à Atenção Primária à Saúde a utilização do mesmo poderia ser incorporada aos processos de trabalho das equipes a fim de contribuir para a qualificação da assistência aos pacientes com doenças crônicas, em especial a hipertensão arterial sistêmica.

Descritores: Atenção Primária à Saúde. Fatores de Risco. Doença crônica

\footnotetext{
1 Trabalho premiado com Menção Honrosa no I Simpósio do Grupo de Estudos Integrado da Universidade de Brasília, Faculdade de Ceilândia

2 Enfermeira. Programa de Residência em Área Profissional da Saúde. Enfermagem em Emergência. Secretaria de Estado de Saúde do Distrito Federal. Brasília, Brasil. Quadra 07, Casa 95, CEP 72425070, Gama Oeste, Distrito Federal. Telefone para contato (61)8149-7270. E-mail: nairapsousa@hotmail.com

3 Professora Adjunta. Faculdade de Ciências da Saúde. Universidade de Brasília. Brasília, Brasil. E-mail: mariafatimasousa@uol.com.br

4 Enfermeira. Secretaria de Saúde do Distrito Federal. Brasília, Brasil. E-mail:djane.elys@yahoo.com.br

5 Doutora em Ciências. Professora Adjunta do Curso de Enfermagem da Universidade de Brasília. Brasília, Brasil.

E-mail: walterlaniasantos@gmail.com

6 Doutorando PPCTS UNB/FCE e Mestre FEN/UFG. Professor Assistente do Curso de Enfermagem da Universidade de Brasília. Brasília, Brasil. E-mail: ramosll@unb.br

7 Doutora em Ciências. Professora Adjunta do Curso de Enfermagem da Universidade de Brasília. Brasília, Brasil.

E-mail: tania.rehem@gmail.com
} 
ABSTRACT: Objective: Stratify the cardiovascular risk in patients with systemic arterial hypertension treated at Regional Health in the Federal District. Method: quantitative approach study with sample of 51 users. It was applied to the sample the Framingham risk score is based on the following predictors: age, LDL (Low Density Lipoproteins) cholesterol, HDL (High Density Lipoproteins) cholesterol, blood pressure, diabetes and smoking. The data were analyzed by means of arithmetic average, standard deviation, absolute and relative frequencies. Results: 43,1\% were low risk, 41,2\% moderate risk and 15,7\% high cardiovascular risk. Conclusion: Although the Framingham Risk Score be mainly used in external scenarios to primary health care, the use of it could be incorporated into the work processes of the teams in order to contribute to the quality of the patient assistance with chronic diseases, especially hypertension.

Key words: Primary Health Care. Risk Factors. Chronic Disease

RESUMEN: Objetivo: Estratificar el riesgo cardiovascular en pacientes con hipertensión arterial sistémica tratada en Regional de Salud en el Distrito Federal. Método: Estudio enfoque cuantitativo con una muestra de 51 usuarios. Se aplicó a la muestra el Escore de Riesgo de Framingham que se basa en los siguientes factores predictores: la edad, el LDL (Low Density Lipoproteins) colesterol, el HDL (High Density Lipoproteins) colesterol, la presión arterial, la diabetes y el tabaquismo. Los datos fueron analizados por la media aritmética, desviación estándar, las frecuencias absolutas y relativas. Resultados: el 43,1\% eran de bajo riesgo, el 41,2\% de riesgo moderado y el 15,7\% de alto riesgo cardiovascular. Conclusión: A pesar de la puntuación del Escore de Riesgo de Framingham ser más utilizado en escenarios externos a la Atención Primaria de Salud, el uso de la misma podría incorporarse en los procesos de trabajo de los equipos con el fin de contribuir a la calificación de la atención a pacientes con enfermedades crónicas, especialmente hipertensión arterial sistémica Palabras clave: Atención Primaria de Salud. Factores de Riesgo.

\section{INTRODUÇÃO}

A Hipertensão Arterial Sistêmica (HAS) acomete 20\% da população brasileira, representando cerca de 20 milhões de indivíduos. A prevalência dessa patologia varia de 5\%, na faixa etária de 18 a 24 anos, a 58\% entre a população com idade superior a 65 anos, e constitui-se fator de risco para as principais causas de morte no Brasil: infarto agudo do miocárdio, acidente vascular encefálico e insuficiência cardíaca. As doenças cardiovasculares relacionadas à HAS representam $7,4 \%$ das internações hospitalares, correspondendo a 13\% (900 milhões de reais ao ano) dos gastos hospitalares do SUS ${ }^{1}$.

Para o enfrentamento dessas questões, o Ministério da Saúde (MS) enfatiza, ente outros aspectos, a importância das equipes multiprofissionais na Atenção Primária à Saúde (APS) frente à prevenção, acompanhamento e controle dos pacientes portadores de hipertensão. Porém, diversas dificuldades ainda persistem no processo de cuidado a esses pacientes, podendo-se destacar a operacionalização do acompanhamento, ou seja, a estruturação da rotina de consultas médicas e de enfermagem². 
Nesse sentido, o MS recomenda a estratificação dos pacientes hipertensos segundo Escore de Risco de Framingham (ERF) para doença cardiovascular. ${ }^{2}$ A estratificação dos indivíduos em subpopulações por riscos permite identificar indivíduos com necessidades de saúde semelhantes que devem ser atendidos com recursos e tecnologias específicos. Além disso, a estratificação de risco está associada à melhor qualidade da atenção à saúde e maior eficiência no uso dos recursos de saúde, já que diminui os erros associados à suboferta de cuidados para portadores de maiores estratos de risco e a ofertas em demasia para aqueles de menores estratos ${ }^{2}$.

Entretanto, a estratificação ainda não está inserida na rotina de trabalho dos centros de saúde, gerando por consequência a oferta de um cuidado que não agrega valor as reais necessidades do indivíduo ${ }^{2}$.

Assim, considerando a magnitude das doenças crônicas não-transmissíveis no Brasil (DCNT), o presente estudo objetiva estratificar o risco cardiovascular de pacientes hipertensos atendidos em centros de saúde. Espera-se dessa forma, contribuir para o enfrentamento das doenças crônicas na atenção primária, mais especificamente com relação às doenças cardiovasculares, de modo que os usuários possam receber um cuidado mais individualizado, gerando por consequência, melhor qualidade de vida, menores índices de internações hospitalares e gastos ao sistema de saúde.

\section{MÉTODOS}

Com abordagem quantitativa o estudo foi realizado na Regional de Saúde do Distrito Federal, em Centro de Saúde da Região Administrativa de Ceilândia, a qual está em processo de implantação da estratificação de risco para pacientes hipertensos. A coleta de dados foi realizada entre os períodos de dezembro de 2013 a fevereiro de 2014. A amostra foi composta por 51 pacientes cadastrados no referido centro de saúde e selecionada de forma não-probabilística por conveniência.

Foram incluídos na amostra os usuários que atendiam, necessariamente, a todos os requisitos abaixo:

$\checkmark$ Homens e Mulheres com idade entre 30 e 74 anos - intervalo estabelecido pelo Escore de Framingham;

$\checkmark$ Cadastro no grupo de Hipertensos desenvolvido no Centro de Saúde;

$\checkmark$ Prontuário eletrônico individual contendo resultados de exames referentes aos níveis de HDL (High Density Lipoproteins) colesterol e LDL (Low Density Lipoproteins) colesterol realizados, no mínimo, no ano de 2013;

$\checkmark$ Assinatura do Termo de Consentimento Livre e Esclarecido (TCLE).

Foram excluídos da amostra os usuários que não atenderam a pelo menos um dos critérios acima definidos. 
Os pacientes foram estratificados segundo o ERF para Doença Cardiovascular (DCV), o qual estima o risco de indivíduos, entre 30 e 74 anos, desenvolverem nos próximos 10 anos um evento cardiovascular maior que inclui o infarto agudo do miocárdio ou morte por determinada causa cardiovascular ${ }^{3}$.

Este escore está proposto como forma de estratificação do risco cardiovascular no protocolo ministerial de Prevenção Clínica de DCV, Cerebrovascular e Renal. O ERF baseia-se em diferentes fatores preditores de risco: idade, LDL colesterol, HDL colesterol, pressão arterial, diabetes e tabagismo. Cada variável possui valores numéricos correspondentes, os quais são específicos para homens e mulheres. Eles devem ser coletados e posteriormente somados para que seja estimado o risco cardiovascular em baixo $(<10 \%)$, moderado (10 a $20 \%)$ e alto $(>20 \%)^{3}$.

A primeira etapa da coleta de dados consistiu na recepção aos pacientes que estavam presentes no Centro de Saúde para a participação na palestra referente ao grupo de Hipertensos. Assim, foi iniciado pela pesquisadora um diálogo que abordava a temática da hipertensão arterial, discutindose assuntos referentes à: definição de hipertensão arterial sistêmica, bons hábitos de vida, formas de tratamento e principais complicações da doença. Após a abordagem dos temas acima referidos, foi inserida no diálogo a temática de Estratificação de Risco e com ela, a explicação dos objetivos e métodos da presente pesquisa. O tempo médio de duração dessa etapa foi de 20 minutos.

A segunda etapa foi iniciada com o convite, aos presentes, para participação na pesquisa, ressaltando-se a necessidade de possuir a idade abrangida pelo escore. Aqueles que aceitaram participar foram convidados a realizar a leitura do TCLE com o auxílio da pesquisadora. Após o entendimento de todos os requisitos pontuados no termo, os mesmos realizaram a assinatura nas duas vias disponíveis.

Procedeu-se então, a análise dos exames referentes à LDL-colesterol e HDL-colesterol, a fim de verificar se ambos estavam inseridos no período determinado para a pesquisa. Todos os exames laboratoriais inseridos no prontuário eletrônico dos pacientes participantes da pesquisa foram realizados no Laboratório Central de Saúde Pública do Distrito Federal (LACEN/DF). Dessa forma, os valores de referência considerados adequados para LDL colesterol foram aqueles $<130 \mathrm{mg} / \mathrm{dL}$ e para HDL colesterol aqueles compreendidos entre 40-60mg/dL, em conformidade com o disposto pela Sociedade Brasileira de Cardiologia ${ }^{4}$.

Após a confirmação desses dados foi realizada, pela pesquisadora, a aferição da pressão arterial - dando início a terceira etapa da pesquisa. Para esse processo, foi utilizado esfigmomanômetro aneróide Premium ${ }^{\circledR}$, previamente calibrado por profissionais em empresa autorizada, o qual possui manguito para a extensão de 18 a 35 centímetros. Além desse equipamento foi utilizado estetoscópio Premium ${ }^{\circledR}$. Essa etapa foi realizada após a participação na palestra a fim de que todos os participantes estivessem em estado de repouso.

A aferição foi realizada em membro superior esquerdo, adotando-se para todos os pacientes a posição sentada $\left(90^{\circ}\right)$ conforme recomendação do Ministério da Saúde ${ }^{3}$. Em relação aos níveis 
pressóricos, os valores foram tratados segundo classificações do Ministério da Saúde ${ }^{2}$. Assim, após a coleta dos valores referentes ao LDL e HDL-colesterol e pressão arterial, os pacientes foram questionados quanto aos dados relacionados a diabetes e tabagismo.

Concluída a etapa de coleta dos dados, foi realizada somatória dos valores correspondentes de cada variável com posterior verificação do correspondente ao Risco Cardiovascular. Assim, o nível de risco encontrado foi registrado no Prontuário Eletrônico de cada paciente e os mesmos receberam a orientação quanto ao significado dos resultados encontrados. Cada participante da pesquisa foi identificado no Instrumento de Coleta de Dados com o número do registro de seu prontuário eletrônico, o que permitirá a realização do acompanhamento necessário pela equipe do centro de saúde.

Os dados foram inseridos em planilha no programa Microsof Excel ${ }^{\circledR}$ e analisados por meio de média aritmética (medida de tendência central), desvio-padrão (medida de dispersão), frequência absolutas (n) e relativas (\%).

Por envolver pesquisa com seres humanos, o presente estudo foi aprovado pelo Comitê de Ética em Pesquisa da Secretaria de Estado de Saúde do Distrito Federal com parecer no 396.042 de 16/09/2013. A pesquisa obteve prosseguimento somente mediante assinatura do Termo de Consentimento Livre e Esclarecido (TCLE) por cada participante da pesquisa, conforme recomenda a Resolução no 466/2012 do Conselho Nacional de Saúde 5 .

\section{RESULTADOS}

A amostra total compreendeu 51 pacientes sendo a maioria $(74,5 \%)$ do sexo feminino com idade média de 59,3 $\pm 9,6$ anos. Do percentual total de pacientes acompanhados, 43,1\% apresentou baixo risco. 41,2\% moderado risco e 15,7\% em alto risco para evento cardiovascular em 10 anos (Tabela $1)$.

Tabela 1 - Distribuição dos pacientes conforme sexo, média de idade e estratos de risco de Framingham. Ceilândia, DF, 2014.

\begin{tabular}{lc}
\hline \multicolumn{1}{c}{ Características } & n (\%) \\
\hline Total de pacientes & $51(100 \%)$ \\
Sexo Feminino & $38(74,5 \%)$ \\
Sexo Masculino & $13(25,4 \%)$ \\
Média Idade (anos) $^{*}$ & $59,5( \pm 9,2)$ \\
Idade (anos) mulheres $^{*}$ & $59,3( \pm 9,6)$ \\
Idade (anos) homens $^{*}$ & $59,9( \pm 8,2)$ \\
$\quad$ Estratos de Risco & \\
$\quad$ Baixo & $22(43,1 \%)$ \\
$\quad$ Moderado & $21(41,2 \%)$ \\
$\quad$ Alto & $8(15,7 \%)$ \\
\hline
\end{tabular}

"média \pm desvio padrão; ${ }^{* *}$ conforme escores de risco de Framingham. 
No escore de baixo risco, $86 \%$ dos pacientes pertenciam ao sexo feminino e apresentavam idade média de 56 \pm 11 anos. Já no escore de moderado risco as mulheres representaram 76,1\% da amostra, exibindo maior média de idade $(62,2 \pm 6,7$ anos $)$ que no escore anterior. $\mathrm{O}$ escore de alto risco, porém, mostrou-se com perfil distinto, pois $62,5 \%$ dos pacientes pertenciam ao sexo masculino, apresentando média de idade de 65 99,1 anos (Tabela 2).

Analisando-se as faixas etárias, independentemente do sexo, têm-se diferentes padrões segundo os três escores obtidos: as faixas etárias compreendidas entre 50 e 69 anos corresponderam a $63,6 \%$ dos pacientes estratificados em baixo risco e os idosos, com idade entre 60 e 69 anos, corresponderam à maioria nos estratos de moderado e alto risco.

A faixa etária mais jovem, de 30 a 39 anos, não possuiu, dentre a amostra selecionada, participantes estratificados nos escores de moderado e alto risco.

Nas faixas etárias de 60 anos e mais, os escores de risco moderado e alto superaram o de baixo risco, panorama que não ocorreu nas idades inferiores a esta, nas quais o escore de baixo foi predominante entre os demais (Tabela 2).

Tabela 2 - Estratos de Risco segundo sexo e faixas etárias. Ceilândia, DF, 2014

\begin{tabular}{|c|c|c|c|c|c|}
\hline Variáveis de Risco & $(\mathrm{n}=22)$ & B a i x o & $\begin{array}{l}\text { Moderado } \\
(\mathrm{n}=\mathbf{2 1})\end{array}$ & $\begin{array}{l}\text { Alto } \\
(\mathrm{n}=8)\end{array}$ & $\begin{array}{l}\text { TOTAL } \\
(\mathrm{n}=\mathbf{5 1})\end{array}$ \\
\hline Sexo Feminino & & 19 & $16(76,1 \%)$ & 3 & $38(74,5 \%)$ \\
\hline Sexo Masculino & $\begin{array}{c}(86 \%) \\
(13,6 \%)\end{array}$ & 3 & $5(23,8 \%)$ & $\begin{array}{l}(37,5 \%)_{5} \\
(62,5 \%)\end{array}$ & $13(25,4 \%)$ \\
\hline \multicolumn{6}{|l|}{ Faixas Etárias (anos) } \\
\hline $30-39$ & & 2 & 0 & 0 & $2(3,9 \%)$ \\
\hline $40-40$ & $(9 \%)$ & 4 & $1(4,8)$ & 0 & $5(9,8 \%)$ \\
\hline $4 \mathrm{~V}=4 \mathrm{9}$ & $(18,1 \%)$ & & $6(28,6 \%)$ & 2 & $15(29,4 \%)$ \\
\hline $50-59$ & $(31,8 \%)$ & & $12(57,1 \%)$ & $(25 \%)_{4}$ & $23(45,1 \%)$ \\
\hline $\begin{array}{l}60-69 \\
70-74\end{array}$ & $\begin{array}{c}(31,8 \%) \\
(9 \%)\end{array}$ & & $2(9,5)$ & $\begin{array}{l}(50 \%) \\
(25 \%)\end{array}$ & $6(11,8 \%)$ \\
\hline
\end{tabular}

A maioria dos pacientes $(56,9 \%)$ apresentou valores não desejáveis para LDL-colesterol. Dentro desse grupo de pacientes, 72,4\% eram do sexo feminino. Além disso, 48,3\% dos indivíduos que apresentaram LDL colesterol alterado foram estratificados no escore de moderado de risco; $27,6 \%$ no de alto risco e $24,1 \%$ no escore de baixo risco. Entre aqueles com níveis adequados de LDL colesterol, a maioria $(68,2 \%)$ foi estratificada no escore de baixo risco e nenhum desses pacientes apresentou estratificação no grupo de alto risco (Tabela 3). Quanto aos valores de HDL colesterol, 90,2\% dos pacientes apresentaram níveis adequados e, desses, 73,9\% correspondiam ao sexo feminino. Dentro desse grupo, $39,1 \%$ obtiveram estrato moderado de risco; $43,5 \%$ baixo risco e $17,4 \%$ alto risco. Apenas 9,8\% dos pacientes apresentaram valores inadequados para HDLcolesterol, apresentando em sua maioria (60\%) estratificação em moderado risco (Tabela 3). 
Tabela 3 - Estratos de Risco segundo LDL-colesterol e HDL-colesterol. Ceilândia, DF, 2014.

\begin{tabular}{|c|c|c|c|c|}
\hline Variáveis de Risco & $\begin{array}{l}\text { Baixo } \\
(n=22)\end{array}$ & $\begin{array}{c}\text { Moderado } \\
(\mathrm{n}=\mathbf{2 1})\end{array}$ & $\begin{array}{c}\text { Alto } \\
(\mathrm{n}=8)\end{array}$ & $\begin{array}{l}\text { TOTAL } \\
(\mathrm{n}=51)\end{array}$ \\
\hline \multicolumn{5}{|l|}{ LDL-colesterol } \\
\hline Adequado & $\begin{array}{r}15 \\
(68,2 \%)\end{array}$ & $\begin{array}{r}7 \\
(31,8 \%)^{7}\end{array}$ & $\begin{array}{l}0 \\
8\end{array}$ & $\begin{array}{r}22 \\
(43,1 \%)\end{array}$ \\
\hline Inadequado & $7(24,1 \%)$ & $\begin{array}{r}14 \\
(48,3 \%)\end{array}$ & $\begin{array}{r}8 \\
(27,6 \%)\end{array}$ & $\begin{array}{c}29 \\
(56,9 \%)\end{array}$ \\
\hline HDL-colesterol & 20 & 18 & 8 & 46 \\
\hline Adequado & $(43,5 \%)$ & $(39,1 \%)$ & $(17,4 \%)$ & $(90,2 \%)$ \\
\hline Inadequado & $2(40 \%)$ & $(60 \%)^{3}$ & 0 & $5(9,8 \%)$ \\
\hline
\end{tabular}

O tabagismo foi relatado por $9,8 \%(\mathrm{n}=5)$ dos indivíduos os quais, em sua maioria ( $80 \%)$, eram do sexo masculino e possuíam média de idade de 59,2 $\pm 11,4$ anos. Desses pacientes, $40 \%$ obteve estrato de baixo risco, $40 \%$ alto risco e $20 \%$ moderado risco. A variável de LDL- colesterol estava alterada em $80 \%$ desses pacientes.

O diabetes mellitus foi relatado por $15,7 \%$ dos pacientes. Desses, $62,5 \%$ pertenciam ao sexo feminino e possuíam idade média de 58,1 $\pm 9,2$ anos. Além disso, 50\% obteve baixo estrato de risco para doença cardiovascular.

Em relação à pressão arterial verificou-se o seguinte panorama: $68,6 \%$ dos pacientes apresentaram níveis adequados, estando relacionados a menores estratos de risco, e, 31,4\% valores inadequados apresentando maiores estratos de risco (Tabela 4). Vale ressaltar que todos os pacientes já haviam sido diagnosticados com HAS e estavam em tratamento na unidade de saúde.

Tabela 4 - Estratos de Risco segundo níveis pressóricos, Ceilândia, DF, 2014.

\begin{tabular}{ccccc}
\hline Variáveis de Risco & $\begin{array}{c}\text { Baixo } \\
(\mathbf{n = 2 2})\end{array}$ & $\begin{array}{c}\text { Moderado } \\
(\mathbf{n = 2 1})\end{array}$ & $\begin{array}{c}\text { Alto } \\
(\mathbf{n = 8})\end{array}$ & $\begin{array}{c}\text { TOTAL } \\
(\mathbf{n = 5 1 )}\end{array}$ \\
\hline Pressão Arterial Adequada & 20 & 12 & 2 & $\begin{array}{c}35 \\
(90,9 \%)\end{array}$ \\
Hipertensão Estágio 1 & 2 & $(57,1 \%)$ & $(25 \%)$ & $(68,6 \%)$ \\
Hipertensão Estágio 2 & $(9,1 \%)$ & $(23,8 \%)$ & $(25 \%)$ & $(15,7 \%)$ \\
Hipertensão Estágio 3 & 0 & $(14,3 \%)$ & $(37,5 \%)$ & $(11,8 \%)$ \\
\hline
\end{tabular}

\section{DISCUSSÃO}

Estudo utilizando ERF para estratificação de risco cardiovascular de usuários atendidos por equipe de Saúde da Família no estado de Alagoas apontou, assim como o presente estudo, para um cenário de predominância do sexo feminino, o qual representou mais de $70 \%$ da amostra ${ }^{6}$. Perfil semelhante foi encontrado por Cavagioni ${ }^{7}$, em estudo com profissionais de saúde que atuavam em atendimento pré-hospitalar em São Paulo, e por Pimenta ${ }^{8}$ com amostra constituída por funcionários 
de uma universidade pública em Minas Gerais.

A maior procura aos serviços de saúde pelo sexo feminino, independente de causa, é evidenciada em vários estudos, justificado, entre outras questões, por pensamentos de fragilidade e/ou vulnerabilidade do sexo masculino ${ }^{9-11}$.

A predominância do escore de baixo risco no presente estudo está em consonância com outros autores $^{6,7,12}$. Este cenário pode está relacionado com o predomínio de níveis pressóricos adequados, os baixos índices de tabagistas e diabéticos e idade média da população estudada inferior a 60 anos. Tais fatores refletem na soma de pontos referentes a cada uma dessas variáveis, gerando por consequência valores correspondentes ao escore de baixo risco.

Nesse contexto, o controle da pressão arterial é um dos desafios encontrados não só pelas equipes de saúde brasileiras, mas por outras equipes de países como Alemanha e Estados Unidos. Nesses países, cerca de 18,5\% e 18\%, respectivamente, da população portadora de HAS e atendida no nível ambulatorial possui sua pressão arterial em níveis controlados. Essa porcentagem é de cerca de $8 \%$ quando se analisa a população da Europa. Índices superiores são encontrados em países como Canadá $(66 \%)$ e Cuba $(40 \%)^{2}$. No caso desse último país, os valores aumentaram consideravelmente quando a população em questão possuía atendimento regular na $\mathrm{APS}^{2}$.

Assim, para pacientes estratificados no escore de baixo são recomendadas, além das consultas anuais com médicos e enfermeiros, medidas de promoção e prevenção em saúde e estímulo para implantação do autocuidado apoiado. A avaliação da capacidade de autocuidado do paciente será influenciada por sua percepção sobre a condição crônica, necessitando, portanto, de avaliação contínua por parte dos profissionais de saúde².

Outra estratégia que pode ser implantada na APS é o cuidado compartilhado, por meio do qual é possível realizar o acompanhamento da situação de saúde dos pacientes de uma forma coletiva. Ou seja, a equipe de saúde convida os pacientes estratificados no mesmo escore de risco para formação de grupos com números determinados. Durante as reuniões, a dinâmica é dada pela exposição de questionamentos e experiências dos próprios pacientes com consequente compartilhamento de conhecimentos. Essa ferramenta permite que o paciente e suas reais necessidades sejam o foco do processo de educação em saúde promovendo assim maior efetividade de ações e fortalecimento de vínculos entre profissionais de saúde e pacientes ${ }^{2,13}$.

Considera-se que tais estratégias de promoção e prevenção em saúde, adotadas pelas equipes de saúde no âmbito da APS, devem atender de forma eficiente às necessidades de saúde dos indivíduos hipertensos. Porém, percebe-se a necessidade de uma visão mais ampliada a cerca da assistência a esses pacientes, tendo em vista a grande complexidade e variedade de fatores que interferem nesse processo. Para isso, é de fundamental importância que cada profissional, seja ele de enfermagem ou não, tenha o compromisso de identificar as necessidades individuais e coletivas da população sob sua responsabilidade, a fim de evitar prescrições demasiadamente generalizadas e desconectadas do contexto ao qual o indivíduo está inserido. 
Assim como no presente estudo, outros obtiveram mesmo padrão de estratificação, ou seja, o risco de evento cardiovascular foi baixo para a maioria dos pacientes, sendo seguido pelos estratos de moderado e alto risco ${ }^{6,7}$.

Nesse sentido, aqueles classificados com menores riscos (baixo e moderado) devem ter sua atenção voltada para atividades de mudanças no estilo de vida e autocuidado. Aqueles em condições de alto risco terão maior concentração de cuidados pela equipe de saúde no âmbito da atenção primária e especializada, mantendo agregadas em seu tratamento tanto a terapêutica medicamentosa quando aquela relacionada a mudanças no estilo de vida ${ }^{2}$.

Há ainda a recomendação do MS de que os indivíduos com escores de moderado risco tenham consultas médicas e de enfermagem com seguimento semestral e os de alto risco possam ser acompanhados trimestralmente. Além disso, todos os pacientes, independentemente dos estratos de risco, devem ter acesso a orientações quanto a mudanças no estilo de vida e participação em ações individuais e coletivas relativas à educação em saúde ${ }^{2,13}$.

Em relação às faixas etárias, o presente estudo, corroborando outros autores, revelou que o aumento progressivo da idade está relacionado em maior participação nos estratos de risco mais elevados para o desenvolvimento de evento cardiovascular no período de 10 anos $^{7,12}$. Sampaio ${ }^{6}$ apontou resultado distinto encontrando predominância de escore de alto risco na população com faixa etária igual ou superior a 50 anos.

No que diz respeito ao tabagismo, os resultados do presente estudo foram semelhantes aos encontrados por Sampaio ${ }^{6}$. Porém, outros trabalhos, com características de amostra distintas, apontaram maior porcentagem de tabagistas no grupo analisado ${ }^{7,8}$. Esse cenário é um recorte do panorama brasileiro que apresenta cerca de $15,1 \%$ da população adulta fumante. Tais dados são expressivos, tendo em vista que o tabagismo está diretamente envolvido, juntamente com outros fatores de risco modificáveis, no processo causal de DCNT como neoplasias de pulmão, bronquite crônica, cardiopatias isquêmicas e doenças cerebrovasculares. Além disso, o tabagismo, em pacientes hipertensos, está relacionado à dificuldade em atingir níveis pressóricos adequados, resistência às drogas anti-hipertensivas e aumento do risco de avanço da insuficiência renal e de complicações cardiovasculares².

Outros estudos também encontraram representação importante de sua amostra com níveis indesejáveis de colesterol. Cavagioni ${ }^{7}$ registrou que $29,2 \%$ dos indivíduos apresentava níveis indesejáveis de LDL-colesterol e 89\% exibia níveis indesejáveis de HDL-colesterol. Sampaio ${ }^{6}$ demonstrou que 69,3\% da amostra apresentava alguma alteração nos níveis de colesterol. Sabese que níveis elevados de colesterol estão relacionados ao aumento do risco de ocorrência de patologias relacionadas ao aparelho circulatório. Além disso, estudos apontaram que cerca de $6,5 \%$ da mortalidade é atribuível ao colesterol, o qual posiciona-se à frente de fatores de riscos como sedentarismo e sexo não-protegido ${ }^{2,14}$.

Em relação às medidas de promoção e prevenção em saúde, recomenda-se a inserção da orientação alimentar no âmbito da APS buscando-se prevenir a ocorrência de excesso de peso e 
alto consumo de gorduras e carboidratos ${ }^{2,13}$.

\section{CONCLUSÃO}

O Escore de Risco de Framingham para estratificação do risco cardiovascular é mais utilizado em cenários externos à APS e com populações de características distintas. Entretanto, a utilização da estratificação de risco poderia ser incorporada aos processos de trabalho das equipes de saúde a fim de contribuir para a qualificação da assistência aos pacientes com doenças crônicas, em especial a hipertensão arterial sistêmica.

É importante ressaltar que na estratificação de risco, pelo fato de um indivíduo ter sido classificado, naquele momento, no estrato de baixo risco não significa, necessariamente, que o mesmo permanecerá neste estrato durante toda a sua vida. O mesmo ocorre com um indivíduo classificado no estrato de moderado ou alto risco. A permanência ou mudança de estrato é influenciada pelos fatores de risco constantes no escore de risco de Framingham, que podem ser traduzidas em necessidades que, se não satisfeitas, podem levar aos processos de adoecimento e/ou morte. Nesse cenário, profissionais e pacientes desempenham papéis fundamentais, em que os primeiros devem compreender tais necessidades e levá-las em consideração durante o acompanhamento ao paciente, superando o perfil predominante de meros prescritores de ações; e os usuários devem seguir no processo de constituírem-se agentes ativos no cuidado à sua saúde.

Este estudo tem como limitações, o fato de utilizar amostra não-probabilística por conveniência, o que diminui a possibilidade de inferir os resultados para o conjunto total de indivíduos. Cabe ressaltar que cada paciente participante da pesquisa recebeu o benefício imediato de orientações relacionadas ao seu estrato risco bem como consulta médica e de enfermagem agendada na própria unidade. Além disso, a divulgação desta metodologia pode favorecer sua incorporação no processo de trabalho de outras que compõem o cenário das unidades básicas de saúde.

Recomenda-se que estudos deste tipo sejam realizados e aprofundados, no sentido de se realizar a Classificação de Risco Cardiovascular Global, onde o Escore de Framingham esteja inserido como um dos fatores a ser avaliado, juntamente com outras variáveis como a avaliação de peso, da circunferência abdominal, do histórico familiar e de outros exames laboratoriais.

Vale destacar ainda, que a participação multiprofissional é fundamental para atenção integral ao paciente com HAS, já que, por ser uma condição crônica multifatorial, demanda um cuidado que envolva esse conjunto complexo de fatores. Dessa forma, a estratificação de risco possibilita a identificação de necessidades e o direcionamento mais adequado de recursos materiais e humanos gerando por consequência uma atenção à saúde mais efetiva, de melhor qualidade e com custo adequado às necessidades apresentadas.

\section{REFERÊNCIAS BIBLIOGRÁFICAS}

${ }^{1}$ Ministério da Saúde (BR). Secretaria de Vigilância em Saúde. Departamento de Análise de Situação de Saúde. Plano de ações estratégicas para o enfrentamento das doenças crônicas não- 
transmissíveis (DCNT) no Brasil [Internet]. Brasília (DF): Ministério da Saúde; 2011 [acesso em 10 de janeiro de 2015]. Disponível em: http://bvsms.saude.gov.br/bvs/publicacoes/plano_acoes enfrent_dent_2011.pdf

${ }^{2}$ Ministério da Saúde (BR). Secretaria de Atenção à Saúde, Departamento de Atenção Básica. Estratégias para o cuidado da pessoa com doença crônica: hipertensão arterial sistêmica [Internet]. Brasília (DF): Ministério da Saúde; 2013 [acesso em 10 de janeiro de 2015]. Disponível em: http://bvsms.saude.gov.br/bvs/publicacoes/estrategias_cuidado_pessoa_doenca_ cronica.pdf

${ }^{3}$ Ministério da Saúde (BR). Secretaria de Atenção à Saúde, Departamento de Atenção Básica. Hipertensão Arterial Sistêmica para o Sistema Único de Saúde [Internet]. Brasília (DF): Ministério da Saúde; 2006 [acesso em 10 de janeiro de 2015]. Disponível em: http://dab.saude. gov.br/docs/publicacoes/cadernos_ab/abcad15.pdf

${ }^{4}$ Xavier HT, Izar MC, Faria Neto JR, Assad MH, Rocha VZ, Sposito AC, et al. V Diretriz Brasileira de Dislipidemias e Prevenção da Aterosclerose. Arq Bras Cardiol [Internet]. 2013 [acesso em 10 de janeiro de 2015 ]; 101 (4 Suppl 1): S1-20. Disponível em: http://www.sbpc.org. br/upload/conteudo/V_Diretriz_Brasileira_de_Dislipidemias.pdf

${ }^{5}$ Ministério da Saúde (BR). Conselho Nacional de Saúde. Resolução No 466 de 12 de dezembro de 2012: aprova as diretrizes e normas regulamentadoras de pesquisas envolvendo seres humanos [Internet]. Brasília (DF): Ministério da Saúde; 2012 [acesso em 10 de janeiro de 2015. Disponível em: http://bvsms.saude.gov.br/bvs/saudelegis/cns/2013/res0466_12_12_2012. html

${ }^{6}$ Sampaio MR, Melo MB, Wanderley MS. Estratificação do Risco Cardiovascular Global em pacientes atendidos numa Unidade de Saúde da Família (USF) de Maceió, Alagoas. Rev Bras Cardiol [Internet]. 2010 [acesso em 16 de fevereiro de 2015]; 23(1):47-56. Disponível em: http:// sociedades.cardiol.br/socerj/revista/2010 01/a2010_v23 n01 05marcussampaio.pdf

${ }^{7}$ Cavagioni L, Pierin AM. Risco Cardiovascular em profissionais de saúde de serviço de atendimento pré-hospitalar. Rev Esc Enferm USP [Internet]. 2012 [acesso em 16 de fevereiro de 2015]; 46(2):395-403. Disponível em: http://www.scielo.br/pdf/reeusp/v46n2/a18v46n2.pdf

${ }^{8}$ Pimenta AM, Kac G, Souza RR, Ferreira LM, Silqueira SM. Trabalho noturno e risco cardiovascular em funcionários de universidade pública. Rev Assoc Med Bras [Internet]. 2012 [acesso em 16 de fevereiro de 2015]; 58(2): 168-77. Disponível em: http://www.scielo.br/pdf/ $\mathrm{ramb} / \mathrm{v} 58 \mathrm{n} 2 / \mathrm{v} 58 \mathrm{n} 2 \mathrm{a} 12$

${ }^{9}$ Gomes R, Rebello LEFS, Nascimento EF, Deslandes SF, Moreira MC. A atenção básica à saúde do homem sob a ótica do usuário: um estudo qualitativo em três serviços do Rio de Janeiro. Cien Saude Colet [Internet]. 2011 [acesso em 10 de janeiro de 2015]; 16(11):4513-21. Disponível 
em: http://www.scielo.br/pdf/csc/v16n11/a24v16n11.pdf

${ }^{10}$ Figueiredo WS, Schraiber LB. Concepções de gênero de homens usuários e profissionais de saúde de serviços de atenção primária e os possíveis impactos na saúde da população masculina, São Paulo, Brasil. Cien Saude Colet [Internet]. 2011 [acesso em 10 de janeiro de 2015]; 16(1 supl 1):935-44. Disponível em: http://www.scielo.br/pdf/csc/v16s1/a25v16s1.pdf

${ }^{11}$ Rehem, TCMSB, Ciosak SI, Egry EY. Ambulatory care sensitive conditions: general hospital of micro-region of São Paulo municipality, Brazil. Texto Contexto Enferm [Internet]. 2012 [cited 2015 January 10]; 21(3):535-42. Available from: http://www.scielo.br/pdf/tce/v21n3/ en_v21n3a07.pdf

${ }^{12}$ Cesarino CB, Borges PP, Ribeiro RC, Ribeiro DF, Kusumota L. Assessment of cardiovascular risk in patients with chronic kidney disease according to Framingham's criteria. Acta Paul Enferm [Internet]. 2013 [cited 2015 January 10] ; 26(1):101-7. Available from: http:// www.scielo.br/pdf/ape/v26n1/en_16.pdf

${ }^{13}$ Ministério da Saúde (BR). Secretaria de Atenção à Saúde, Departamento de Atenção Básica. Estratégias para o cuidado da pessoa com doença crônica [Internet]. Brasília (DF): Ministério da Saúde; 2014 [acesso em 10 de janeiro de 2015]. Disponível em: http://bvsms.saude.gov.br/bvs/ publicacoes/estrategias_cuidado_pessoa_doenca_cronica_cab35.pdf

${ }^{14}$ Ministério da Saúde (BR). Secretaria de Vigilância em Saúde, Secretaria de Gestão Estratégica e Participativa. Vigitel Brasil 2010: vigilância de fatores de risco e proteção para doenças crônicas por inquérito telefônico [Internet]. Brasília (DF): Ministério da Saúde; 2011 [acesso em 10 de janeiro de 2015]. Disponível em: http://bvsms.saude.gov.br/bvs/publicacoes/ vigitel_2010.pdf

Artigo apresentado em 30-09-15 Artigo aprovado em 25-02-16 Artigo publicado no sistema em 28-03-16 